\title{
TESTICULAR CANCER
}

\section{Long-term outcomes of single BEP cycle}

Adjuvant treatment with one cycle of bleomycin, etoposide and cisplatin (BEP) seems to be a safe alternative to two cycles in patients with high-risk clinical stage I (CS I) nonseminomatous germ cell tumours (NSGCT), say researchers.

Treatment of patients with CS I NSGCT remains a contentious issue. Two cycles of $\mathrm{BEP}$ are recommended for patients at high risk of relapse but concerns exist about toxic effects such as impaired fertility, cardiovascular morbidity, neuropathy and renal toxicity.

Vidal et al. enrolled 44 patients with high-risk CS I NSGCT with vascular invasion and/or $>50 \%$ embryonal cell carcinoma. Patients received one cycle of adjuvant BEP and were followed up for a median of 186 months with a primary end point of rate of relapse. Four patients were excluded from the analysis.

During follow-up, one patient had a pulmonary relapse and died of pulmonary distress syndrome following three additional cycles of BEP. Three patients presented with a metachronous contralateral testicular tumour and three patients developed a second malignancy. After additional treatment, these patients did not relapse during the follow-up

period. Adverse effects included intermittent tinnitus in three patients and peripheral polyneuropathy in one.

"Recurrence rate seems as low as in earlier trials where two adjuvant cycles of BEP were given," say the authors."The advantage of the modified BEP schema, as used by us, is that it lasts only 3 days instead of 5 days. If our promising results are confirmed by other centres one cycle of adjuvant BEP should become a first-line standard in high-risk CS I NSGCT patients."

Rebecca Kelsey

Original article Vidal, A. D. et al. Long term outcome of patients with clinical stage I high-risk nonseminomatous germ cell tumors 15 years after one adjuvant cycle of bleomycin, etoposide and cisplatin chemotherapy. Ann. Oncol. doi:10.1093/annonc/mdu518 